\title{
Real-Time Experimentation and Analysis of Wifi Spectrum Utilization in Microwave Oven Noisy Environment
}

\author{
Yakubu S. Baguda \\ Information System Department, Faculty of Computing and Information Technology, \\ King Abdulaziz University, Rabigh, Saudi Arabia.
}

\begin{abstract}
The demand for broadband wireless communication in home and office has been increasing exponentially; thus, the need for reliable and effective communication is very crucial. Both theoretical and experimental investigations have clearly shown that electromagnetic radiation from external sources such as microwave oven (MWO) has detrimental impact on the wireless medium and the media content. Therefore, this drastically degrade the signal strength in wireless link and consequently affects the overall throughput due to noise and interference. This experimental study is primarily aimed at critically analyzing and evaluating the impact of electromagnetic radiation on spectrum utilization under different experimental scenarios. The experimental results clearly show that electromagnetic noise radiation from microwave oven can seriously affect the performance of other devices operating in 2.4GHz frequency band, especially, delay sensitive applications and services.
\end{abstract}

Keywords-Electromagnetic radiation; microwave oven; spectrum utilization; bandwidth; ISM band; signal strength; throughput; wireless channel

\section{INTRODUCTION}

Wireless communication is increasingly becoming popular and widely used in recent years due its flexibility, scalability and low cost of deployment. It can be potentially applicable in health, public, and commercial sectors. More importantly, it has been used in home environment and hence its potential applications cannot be over emphasized. The $2.4 \mathrm{GHz}$ frequency band is primarily dedicated for industrial, scientific and medical (ISM) usage [1], [2]. Hence the electromagnetic radiation from other equipment operating in the same frequency band could cause interference and subsequently degrades the performance of the wireless network. Cellular phones and microwave ovens are the most common household appliances operating in the frequency band which can electromagnetically radiates noise, and it interferes with other devices. The fact that IEEE802.11b uses the $2.4 \mathrm{GHz}$ frequency band [10], there is every tendency that ISM equipment can reduce the signal strength of the transmitted radio signal over wireless LAN. In [3], [11]-[14], the effective dynamic spectrum utilization for the future wireless networks has been described. The electromagnetic radio frequency (RF) power radiated from microwave oven can cause loss data or connectivity in wireless networks [4], [5]. The impact of some devices on WiFi networks has been studied in [6].
Several theoretical and experimental works related to microwave ovens interference in wireless network have been conducted in order to evaluate and analyze the overall performance of the network [7]. Investigating such phenomenon will assist tremendously in designing efficient strategies on how to mitigate the impact of the interference caused by microwave oven radiation on signal quality. Indeed, this is extremely important with rapid growth in demand for different application over wireless network. Most of the devices and gadget has the capability to seamlessly stream multimedia wirelessly through WLAN at home or office. Microwave oven radiation can reduce the network performance and wireless devices attached to it. By critically analyzing the impact of noise generated by microwave oven on the network and content, adequate provision and network planning can be made by network administrators in order to achieve optimal performance.

Both stochastic and empirical models have been developed using amplitude probability distribution (APD) to model the microwave oven EM radiated noise for simulation purpose. In [1], bit error rate (BER) has been used to evaluate the noise generated by the microwave oven. Higher bit error rate can eventually lead to increase in packet error rate (PER) and it can be computed from the BER. Some researchers used stochastic model to mimic the noise in the wireless link. All these approaches are primarily developed for theoretical analysis of microwave oven noise. In this work, practical noise pulse generated by microwave oven is considered in the experimentation in order to exactly quantify and evaluate its detrimental impact. The fact that large amount of power is released by microwave oven when compared to wireless LAN devices, the performance of wireless devices need to be investigated while microwave oven is actively ON. In [8], [9], it has been clearly indicated that the performance of wireless network can be greatly affected by microwave oven noise. In this paper, real-time experimentation has been conducted to critically evaluate and analyze the interference from external sources which affect the channel quality, throughput and the spectrum utilization based on the real world electromagnetic interference source. There is need to clearly understand and characterize the dynamic nature of interference in $\mathrm{WiFi}$ networks due to their significant role in today's communication and networking industry. Undoubtedly, this will eventually leads to the development of highly efficient 
and effective strategies to mitigate the interference in $\mathrm{WiFi}$ networks.

Section 2 mainly focuses on the effect of microwave ovens on wireless LAN devices from both theoretical and numerical perspectives. Section 3 discusses the experimental procedure and measurement setup used to conduct the experiments. Section 4 discusses the results from the experiments. Finally, conclusions are enumerated in Section 5.

\section{ELECTROMAGNETIC RADIATION EFFECTS}

The electromagnetic energy release by microwave oven can be disastrous to other devices and application using the same ISM band - it can eventually increase the bit error rate and consequently can lead to incessant packet lost. For example, multimedia applications are sensitive to noise and delay which can cause errors while decoding the media content at destination. The degradation of the multimedia content due to EM radiation has not been fully exploited and requires more understanding of the impact of electromagnetic radiation on wireless networks.

\section{A. Theoretical and Experimental Background}

It has experimentally been proved that the power density of microwave oven does not exceed up to $1 \mathrm{~mW} \mathrm{~cm}^{-2}$ from the surface where it radiates the noise energy. Based on statistics, it can be assumed that only $50 \%$ of microwave ovens used today emit less than $0.062 \mathrm{~mW}$ per $\mathrm{cm}^{2}$.

The interference between microwave oven noise and signal transmitted by wireless device can be model as shown in Fig. 1. The amplitude of the pulse generated as noise could be model theoretically. It is very obvious that the amplitude and frequency varies with the source voltage. The mathematical expression representing the noise is described in (1). The model is mainly based on the assumption that both the frequency and amplitude of the noise pulse directly varies with the source voltage variation. Fig. 1 shows a typically model illustrating the effect of noise generated $A(t)$ by microwave oven on transmitted signal. Therefore, the noise pulse model can be represented diagrammatically as follows.

The electromagnetic noise $\mathrm{A}(\mathrm{t})$ generated by the microwave oven can be modeled and represented mathematically as shown in (1):

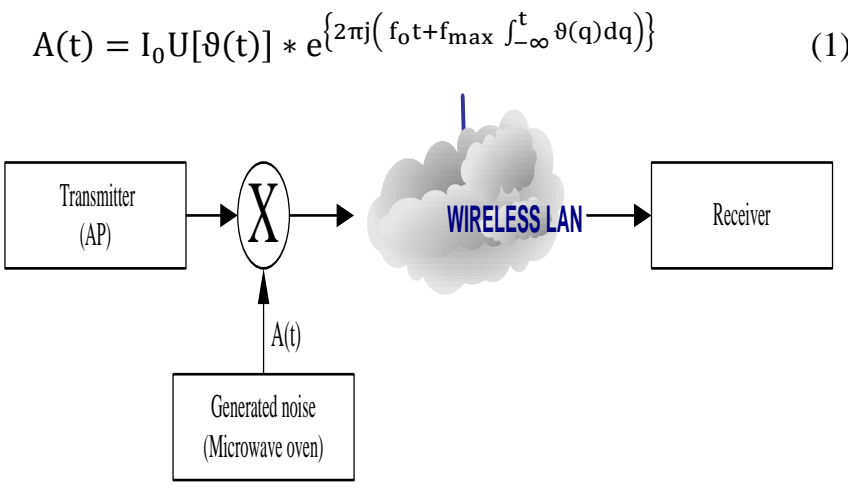

Fig. 1. Model of interference between microwave oven noise and wireless LAN.
Where, $f_{o}$ is the carrier frequency and $f_{\max }$ represents the maximum frequency of deviation.

The amplitude of the pulse radiated is determined by $I_{0}$ and its phase is assumed to be distributed uniformly in order to simplify the problem. As seen from (2), the value of $U$ is a function of $\vartheta$ and it depends on the threshold voltage $\vartheta_{o}$ value.

$$
\begin{aligned}
& U(\vartheta)=\left\{\begin{array}{l}
\vartheta, \text { for } \vartheta \geq \vartheta_{o} \\
0, \text { for } \vartheta<\vartheta_{o}
\end{array}\right. \\
& \vartheta(t)=\left\{\begin{array}{r}
\cos \left(2 \pi f_{v}\right), \text { for transformer type } \\
\cos \left(2 \pi f_{v}\right) \cos \left(2 \pi f_{s}\right), \text { for inverter type }
\end{array}\right.
\end{aligned}
$$

Where, $f_{v}$ and $f_{c}$ represents the A.C. supply and inverter switching frequency respectively as it has been described in (3).

$$
\mathrm{f}(\mathrm{t})=\mathrm{f}_{0}+\mathrm{f}_{\mathrm{m}}(\vartheta(\mathrm{t})), \quad \vartheta(\mathrm{t}) \geq \vartheta_{\mathrm{o}}
$$

The noise generated by microwave oven can be categories as band-limited noise and it can be represented mathematically as shown in (5) below:

$$
N(t)=\int_{-\infty}^{\infty} h(\tau) n(1-\tau) d \tau
$$

Where, $h(\tau)$ represents the complex impulse response for the filter.

\section{MATERIALS AND METHODS}

\section{A. EM Microwave Radiation Source}

The experimental work has been conducted using PANASONIC $^{\circledR} \quad(\mathrm{NN}-\mathrm{S} 215 \mathrm{MF})$ microwave oven with the specifications described in Table I. The microwave oven output power is around 800 watts and it operates at the frequency of $2.45 \mathrm{GHz}$. It is used in the experiment as a source for generating the electromagnetic noise which interferes with other devices operating in the ISM frequency band. Basically, the magnetron in the microwave oven generates RF energy when the voltage exceeded the threshold value. The driving voltage is produced by AC supply source is fed to step-up transformer or inverter depending on the microwave oven type. Consequently, it generates RF pulse within the $2.4 \mathrm{GHz}$ band at the frequency of the supply main or switching frequency of the inverter. The description about the microwave oven used for the experimental work is shown in Table I.

TABLE I. MICROWAVE OVEN DESCRIPTION

\begin{tabular}{|l|l|l|}
\hline $\begin{array}{l}\text { Microwave } \\
\text { Oven Type }\end{array}$ & Description \\
\hline \multirow{4}{*}{$\begin{array}{l}\text { PANASONIC } \\
\text { NN-S215MF }\end{array}$} & Microwave oven type & Transformer / Inverter \\
\cline { 2 - 3 } & Input power & $5.4 \mathrm{~A}, 240$ volts AC supply \\
\cline { 2 - 3 } & Output & $800 \mathrm{w}$ \\
\cline { 2 - 3 } & Frequency & $2.45 \mathrm{GHz}$ \\
\cline { 2 - 3 } & AC mains frequency & $50 \mathrm{~Hz}$ \\
\hline
\end{tabular}


The impact of electromagnetic noise generated at the frequency of $2.45 \mathrm{GHz}$ was constantly monitored and analyzed. A spectrum analyzer and wire shark were used to keep track of variation in signal strength as the distance between the access point (AP) and microwave oven is adjusted. The AP has been maintained at the same position throughout the experiment.

The AP is used in experimentation to serve as the medium through which devices can connect to the network. External radiation from the microwave oven was used to study the variation in signal transmitted over the medium. When the microwave oven is close to the AP, the signal strength reduces due to the interference signal generated by the oven. At every distance, the signal strength is measured using the spectrum analyzer, and PC equipped with wireshark and Hand Held Software Tool (HHS). The signal strength of the channel 6 to 11 within the ISM band has been evaluated to determine the effect of the radiation source. It is very obvious that channel 11 will be affected more when compared to the other channels. This is because of the fact that the microwave frequency of operation falls within its frequency range.

Table II shows the description of the AP used to conduct the experimentation. The AP operates within the frequency range of $2.4 \mathrm{GHz}$ to $2.487 \mathrm{GHz}$ which eventually covers the frequency of operation for the microwave oven. The electromagnetic interference (EMI) and susceptibility of the AP are according to FCC Part 15.107 and 15.109 Class B.

The spectrum analyzer has been set to monitor the frequency range of $100 \mathrm{KHz}$ to $3 \mathrm{GHz}$ and can be able measure the power between the ranges $-110 \mathrm{dBm}$ to $20 \mathrm{dBm}$. More detail about the spectrum analyzer has been presented in Table III.

TABLE II. ACCESS POINT DESCRIPTION

\begin{tabular}{|l|l|l|}
\hline Access Point Type & \multicolumn{2}{|l|}{ Description } \\
\hline \multirow{4}{*}{$\begin{array}{l}\text { CISCO } \\
\text { AERONET 1200 }\end{array}$} & Antenna & $2.2 \mathrm{dBi}$ \\
\cline { 2 - 3 } & Input power & $4.75 \mathrm{w}$ \\
\cline { 2 - 3 } & Typical range & $121.9 \mathrm{~m}$ \\
\cline { 2 - 3 } & Frequency & 2.400 to $2.497 \mathrm{GHz}$ \\
\cline { 2 - 3 } & Temperature & -4 to $131^{\circ} \mathrm{F}\left(-20\right.$ to $\left.55^{\circ} \mathrm{C}\right)$ \\
\cline { 2 - 3 } & EMI and & FCC Part 15.107 and \\
& Susceptibility & 15.109 Class B \\
\hline
\end{tabular}

TABLE III. SPECTRUM ANALYZER DESCRIPTION

\begin{tabular}{|l|l|l|}
\hline Analyzer & Description \\
\hline \multirow{4}{*}{$\begin{array}{l}\text { Spectrum Master } \\
\text { (MS2711D) }\end{array}$} & Frequency range & $100 \mathrm{kHz}$ to $3.0 \mathrm{GHz}$ \\
\cline { 2 - 3 } & Frequency Accuracy & $\begin{array}{l} \pm 10 \mathrm{~Hz}, 99 \% \text { confidence } \\
\text { level }\end{array}$ \\
\cline { 2 - 3 } & Measurement Range & $+20 \mathrm{dBm}$ to $-110 \mathrm{dBm}$ \\
\cline { 2 - 3 } & Channel Power & $\begin{array}{l} \pm 1 \mathrm{~dB} \text { typical }( \pm 1.5 \mathrm{~dB} \\
\max )\end{array}$ \\
\cline { 2 - 3 } & Temperature & -10 to $+50^{\circ} \mathrm{C}$ \\
\cline { 2 - 3 } & $\begin{array}{l}\text { Adjacent channel } \\
\text { power accuracy }\end{array}$ & $\pm 0.75 \mathrm{dBc}$ \\
\hline
\end{tabular}

\section{B. Measurement Setup}

In order to experimentally evaluate and analyse the impact of microwave oven electromagnetic radiation on the transmission medium, there is need to determine the characteristic of microwave oven interference and ultimately determine its detrimental impact on spectrum utilization and wireless network performance. The experiment was conducted in an indoor environment which is typical to normal home setup. A single microwave oven was used in experimentation. Only the personal computer is connected to the network and all other devices have been disconnected from the network in order to effectively monitor the network condition. Fig. 3 shows the setup used for the experiment. The equipment used for the experiment includes spectrum analyzer, Cisco Aeronet 1200 AP, microwave oven and personal computer equipped with wireshark software. The wireshark software plays an integral role in networks by capturing and analyzing packets within an environment it has been set to monitor. More importantly, it can be used in monitoring and troubleshooting networks from bits up to the packets level. This tool provided all the necessary information about the network and it has been used to critically examine the network performance under different network scenarios.

The spectrum master has been designed mainly to monitor, measure and analyze signals. It has the capability to measure in-band interference, transmit spectrum analysis, antenna isolation and cell area interference. More importantly, the spectrum analyzer has been used to determine amount of radio energy within the ISM band. In order to track the impact of the microwave oven radiation, channel 6-11 of the IEEE802.11b was monitored using spectrum analyzer and readings were taken at different distances. This is mainly due to the fact the MWO operates within the frequency range of $2.437 \mathrm{GHz}$ to $2.462 \mathrm{GHz}$ as shown in Fig. 2. The bold line in between $2.437 \mathrm{GHz}$ and $2.462 \mathrm{GHz}$ frequencies shows the frequency at which the microwave operates.

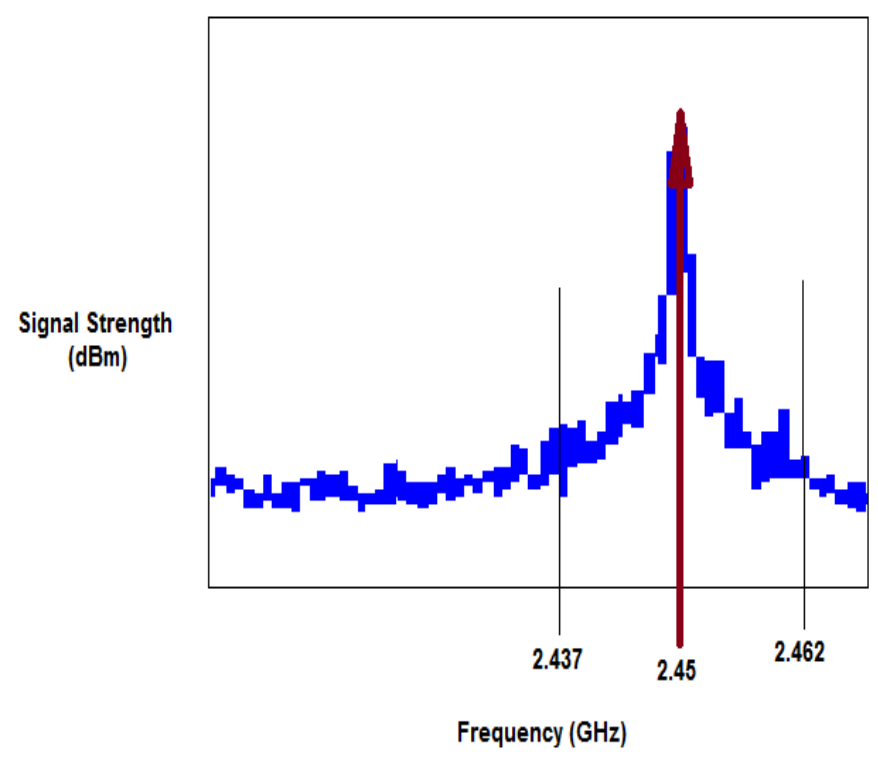

Fig. 2. Microwave oven operating frequency and study range. 


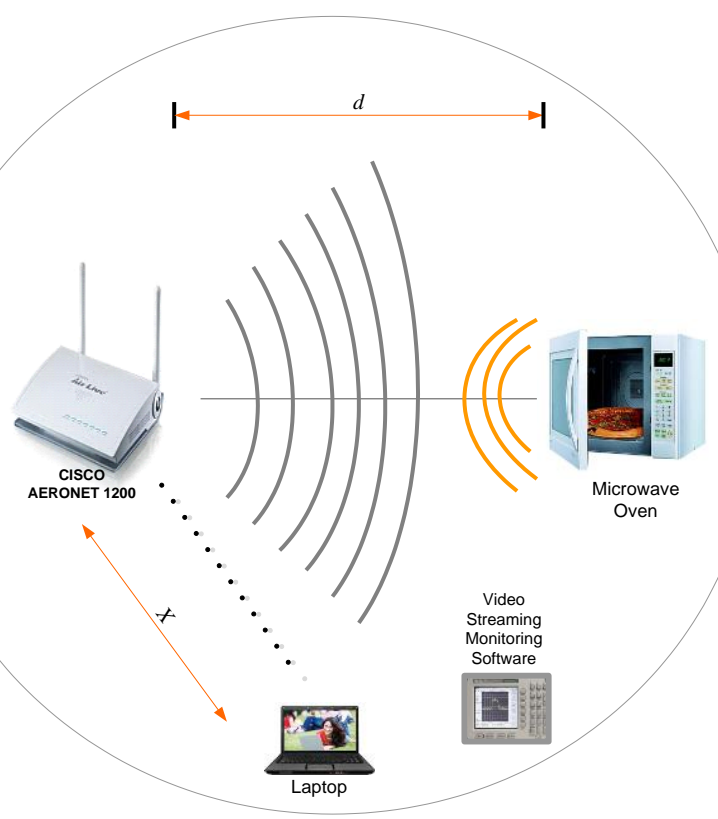

Fig. 3. Experimental setup.

As it has been mentioned earlier, microwave oven is a major source of electromagnetic radiation noise in ISM band. Their fundamental principle of operation is based on generation of microwave energy through the use of magnetron tubes. Interestingly, the microwave oven operates at the center frequency of the ISM band (i.e. $2.45 \mathrm{GHz}$ ). Fig. 3 illustrated how the experimentation was conducted in order to observe how the microwave operation affects the spectrum under different scenarios. The proximity of the devices was a key fundamental issue to monitor in the experimentation.

The available channels were constantly monitored as the distance $d$ and $x$ are varied in order to detect the variation in signal strength and throuhgput as well. The fact that the position of the AP is fixed throughout the experiment while the microwave oven and spectrum analyzer position are changed intermittently based on the experimental scenario needed. The distance between the AP and microwave oven is varied from $2 \mathrm{~m}$ to $10 \mathrm{~m}$ at an interval of $2 \mathrm{~m}$. This is mainly to verify the detrimental impact of the microwave oven radiation on the wireless channel which eventually reduces the spectrum utilization, and consequently it leads to an increased in bit error rate and packet loss within the frequency band.

\section{MEASUREMENT ResUlts AND ANALYSIS}

In the previous section, the procedure on how the experiment was conducted has been discussed. The analysis of the results obtained from the measurements will be covered in this section. The experimental data was collected using spectrum analyser and the wire shark program. Fig. 4 shows the experimental arrangement used for the experimentation. The wireshark software is used to thoroughly check the wireless channels under scrutiny based on the channel signal strength and interefence. The network information captured by the spectrum analyzer was downloaded, and processed by the HHS tool as shown in Fig. 4.

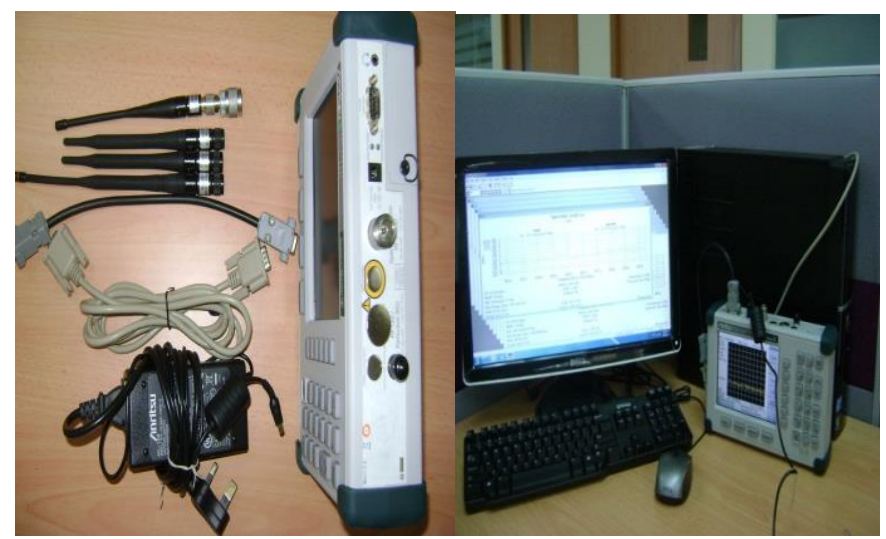

Fig. 4. Mesurement equipment and Tools.

Initially, the experiment was conducted in an enviroment where there is no external interference from any source of interference. Measurements were taken at different distance $(2,4,6,8,10 \mathrm{~m})$ between the AP and PC, and the procedure is repeated 5 times in order to determine the average of the readings taken. Again, the same procedure was repeated when the microwave oven has been switched ON. Subsequently, comparison was made between when there is no interference and the scenario when there is interference from external source (MWO). In both cases, the signal strength, throughput, noise traffic and utilization of the network has been recorded.

Based on the experimental setup in Fig. 3, the distance d between microwave oven and AP was set to a fixed value. The spectrum analyzer monitors the frequency band as the distance between the AP and the microwave oven is adjusted. The signal strength decreases as the proximity between the microwave oven and AP decreases. The network throughput has been used as a metric to measure the performance of the network when subjected to external interference. It is primarily achieved by using the wire shark software to critically analyze the network performance in terms of parameters such as network traffic under different experimental scenarios. Interestingly, it has enabled us to analyze packets individually as the traffic flows through the network.

\section{A. Bandwidth Utilization}

The bandwidth utilization has been considered as a metric to measure the performance of the network when subjected to external interference. In order to effectively utilize the network resources, the interference from external sources should be mitigated. The spectrum utilization is closely monitored using the wire shark software since it has been installed in the PC which was wirelessly connected to the AP. The bandwidth utilization has been observed when the microwave oven is switched ON and OFF as well. The PC in which the wire shark software is installed has been placed at different distance $(2,4,6,8$ \& 10m) from the WiFi transmitter (AP). The entire spectrum is monitored in order to analyze the performance of the network based on the aforementioned parameter. 


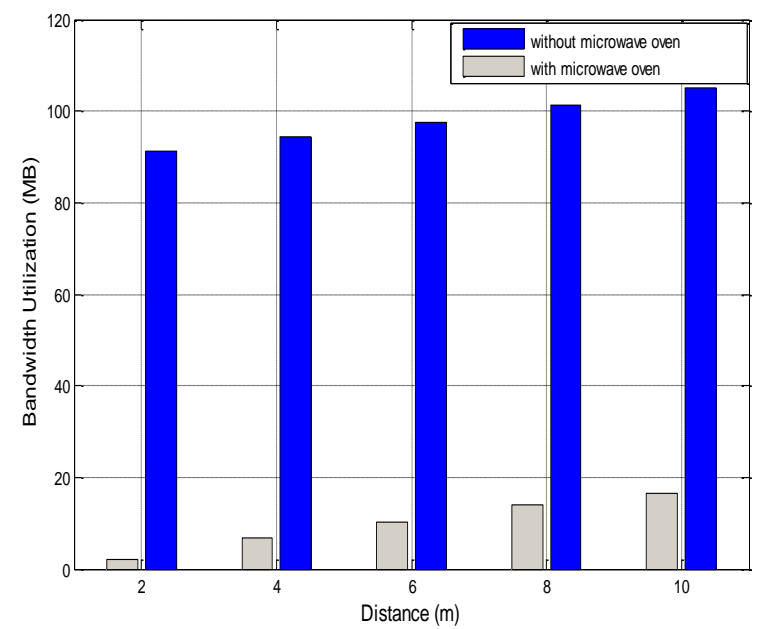

Fig. 5. Bandwidth utilization.

As can be noticed from Fig. 5 that the total traffic level is low when the microwave oven is ON compared to a situation when it is OFF. This is mainly due to the fact that the connectivity between the $\mathrm{PC}$ and $\mathrm{AP}$ is poor when the radiation source is at close proximity. As the monitoring device moves far away from the external interference source, the wireless spectrum could be assessed better and it leads to an increment in the network traffic and vice visa. In a nutshell, the bandwidth utilization is very much high when the PC has been placed far away from the microwave oven. It is obvious that the bandwidth utilization is less when the AP is within close proximity to the microwave. It is primarily due to the fact the external radiation from the microwave oven will destabilize the spectrum and eventually leads to low bandwidth utilization.

\section{B. Signal Strength}

Fig. 6 illustrated the performance of the WiFi network in terms of received signal strength. The received signal strength has been used as a metric to measure the network performance when the microwave oven is ON and OFF as well. Due to detrimental impact of the external radiation from the microwave which reduces the strength of the signal radiated from the AP as it travels along the medium. This adverse effect can be noticed from Fig. 6 which clearly shows that the received signal strength has reduced tremendously with the introduction of microwave oven onto the network. This is primarily due to the fact the MWO operates within the same frequency with the other device connected to the WiFi network. When the MWO is switched ON, the received signal strength reduces with increase in distance from the AP.

The ability to receive signal with high strength depend on many factors, but it is very obvious that the noise generated in the medium which the signal traverse play significant on the received signal strength. Interference from the external sources will cause detrimental impact to the signal quality and channel as well. It can be seen from Fig. 6 that the signal strength decreases with increase in distance. The signal strength varies from $-87 \mathrm{dBm}$ to $-96 \mathrm{dBm}$ as the distance was adjusted from $2 \mathrm{~m}$ to $10 \mathrm{~m}$ in a noisy microwave oven environment. Also, it can be noticed that the signal strength varies from $-18 \mathrm{dBm}$ to $-38 \mathrm{dBm}$ for the same distance, but the microwave oven has been switched off completely. Through the experimentation, it can be noticed that the rate at which the signal strength decreases is much higher in a noisy microwave oven environment when compared to an environment which is not subjected to external interference. In a nutshell, the microwave oven operation within an environment which utilizes wireless connection to communicate and transmit data has detrimental impact on the received signal strength. Non WiFi devices hinder the performance of the WiFi network and ultimately cause incessant packets loss and interference in the network. Developing new techniques on how to handle the problem above will assist greatly in ensuring effective and reliable communication over wireless networks.

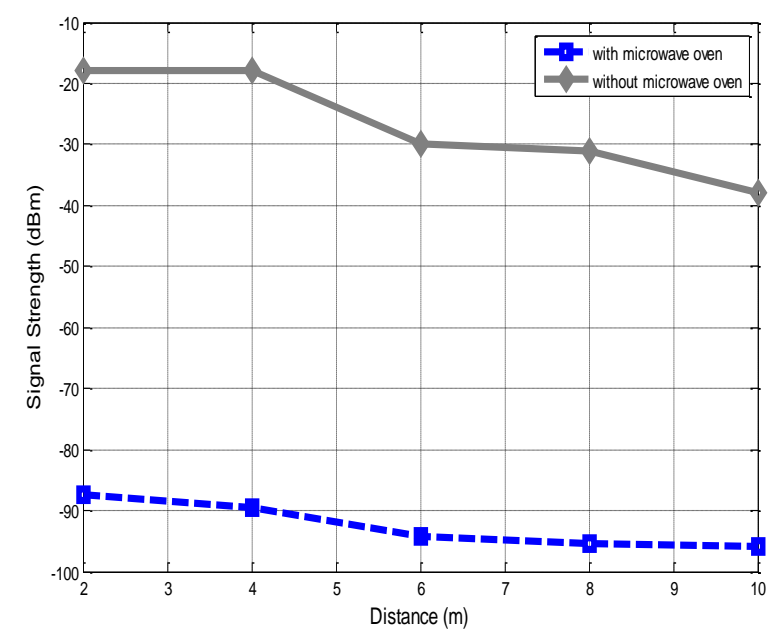

Fig. 6. Signal strength at different proximities.

\section{Network Throughput}

The network throughput has been examined in order to evaluate the impact of microwave oven operation on $\mathrm{WiFi}$ network throughput. The experimental approach used to collect the result is based on the scenario shown in Fig. 3. The impact of microwave oven on the throughput in terms of bits per second (bps) is measured to determine the network the performance under different scenarios. Table IV shows the average value of the throughput captured at different distance when the microwave oven is activated $\left(\mathrm{T}_{\mathrm{ON}}\right)$ and deactivated $\left(\mathrm{T}_{\mathrm{OFF}}\right)$ as well. It very clear that the throughput reduces as the $\mathrm{PC}$ is closed to the microwave oven due its negative influence on the number of bits transmitted per second.

TABLE IV. ACHIEVABLE NETWORK THROUGHPUT WITH AND WITHOUT MiCROWAVE OVEN

\begin{tabular}{|c|c|c|c|}
\hline \multirow{2}{*}{ Distance (m) } & \multicolumn{2}{|c|}{ Throughput (Kbps) } & \multirow{2}{*}{$\zeta(\%)$} \\
\hline & $T_{O N}$ & $T_{O F F}$ & \\
\hline 2 & 395.7 & 566.5 & 30 \\
\hline 4 & 562.8 & 695.1 & 18 \\
\hline 6 & 615.5 & 737.3 & 16.5 \\
\hline 8 & 728.05 & 819.6 & 11.2 \\
\hline 10 & 1000.1 & 1122.1 & 10.8 \\
\hline
\end{tabular}


The percentage deviation $(\zeta)$ in throughput when the MWO is ON and OFF can be computed using (6) as shown below:

$$
\zeta=\left(\frac{T_{O F F}-T_{O N}}{T_{O F F}}\right) \times 100
$$

$\zeta$ is the percentage change in throughput when the microwave is active and deactivated. It shows the percentage at which the overall network throughput drops by juxtaposing $\mathrm{T}_{\mathrm{ON}}$ and $\mathrm{T}_{\mathrm{OFF}}$ at different distance. In order to determine by how much the throughput reduce as a result of microwave oven presence within the wireless environment, $\zeta$ is employed to quantify the rate at which the throughput as the distance increases. (6) has been used in computing the value of $\zeta$ under different scenario. The key fundamental issue is to compare the throughput when the microwave oven is activated and deactivated at the same position starting from $2 \mathrm{~m}$ to $10 \mathrm{~m}$. For instance, at $2 \mathrm{~m}$ distance, it is clear that the microwave oven operation greatly affects the throughput of the network. Even though the PC has close proximity to the AP but due to intense radiation from the external source, it immensely reduces the achievable throughput. Indeed, it can be notice that the achievable throughput increases with increase in distance away from the activated microwave oven and reverse is the case when the microwave oven is inactive. The detrimental impact caused as a result of noisy microwave oven environment and distance on throughput can be observed from the graph shown in Fig. 7.

As it has been mentioned already that the need to study the impact of this phenomenon on network performance will lead way toward developing scheme to effectively deals with the adverse impact of the devices on parameters such as throughput, packet loss and bandwidth utilization. This experimental work has examined the impact of radiation on wireless environment, and later much effort and resources would be dedicated to mitigation strategies to reduce the adverse effect of these home devices on the performance of WiFi. Using cognitive radio scheme, it will intelligently detect and determine the best frequency band to operate. This will reduce the incessant packet loss and increase the throughput. More importantly, the network performance will be enhanced.

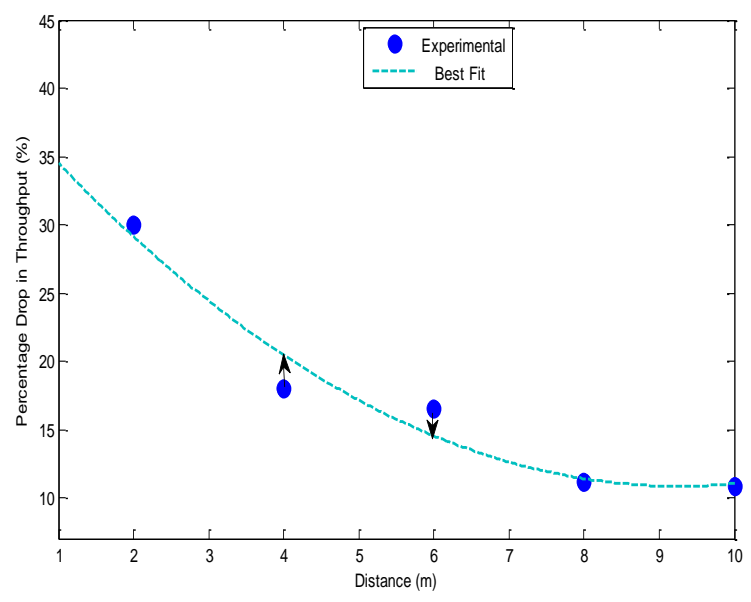

Fig. 7. Percentage in throughput drop with increase in distance.
In this work, only one source of radiation and user has been considered, but there is need to consider multi-users environment which mimic typical real world scenario. There is need to consider different non WiFi devices operating within the frequency and evaluate their detrimental impact on the network performance. More importantly, it would help in designing schemes to address the external radiation and interference problem in wireless network. In our future works, much emphasis will be place on how to significantly improve the network capability by detecting the radiation from non WiFi devices and switching to other frequency band can improves the network performance and ensures more seamless network connectivity. The work will be based on cognitive radio approach in conjunction with other optimization algorithms to enhanced searching ability.

\section{DISCUSSION}

Having examined the problem of external interference within the WiFi networks, the need for highly sophisticated scheme to intelligently mitigate the problem is very crucial and important. Understanding the nature of the interference could dramatically improve the performance of the network since can led to the development effective and efficient solutions to mitigate the interference from the external sources.

It is very obvious that there is dramatic need for efficient utilization of spectrum in order to meet with increasing demand for bandwidth and to efficiently mitigate the bottlenecks which reduces the performance of the network. It is challenging to efficiently mitigate the interference form external sources but there is need to dynamically adapt with environmental changes in a noisy microwave environment in order to achieve efficient spectrum utilization and management. Cognitive radio is a promising technology to ensure effective spectral utilization and management in wireless system. The cognitive radio transmission should be properly controlled such that the disturbance of the primary network is within acceptable limit. More research effort is needed for distributed power control in multi-user cognitive radio network to ensure that the power radiated from different sources does not significantly affect the network performance. An intelligent network management scheme will ensure efficient spectrum utilization, coverage and fairness amongst multiple secondary users. Most of the interference aware approaches focused on wireless devices interference [15]-[18], but more emphasis need to be place on non WiFi devices which eventually affect the network performance and it subsequently effects the applications and services as well.

\section{CONCLUSION}

In this paper, an experimental investigation of effects microwave oven noise has been presented in order to evaluate the electromagnetic radiation impact on WiFi spectrum band. An experimental investigation is conducted to analyze and quantify the performance degradation in link quality and spectrum utilization of wireless channels in a noisy microwave oven environment. This is extremely important as the usage of microwave oven and wireless technology become prevalence at home and offices. The electromagnetic radiation noise impact is verified experimentally based on the signal strength 
and the proximity of the microwave oven to the access point. The proximity of the microwave oven to the device plays an integral role because it determines the amount of distortion to be anticipated by the wireless networks. Our future work will primarily focus in developing an intelligent scheme to cooperatively manage the WiFi spectrum with low packet loss and high throughput while at the same time ensuring coexistence among devices connected to the network.

\section{ACKNOWLEDGMENT}

The author would like to thank all those who contributed toward making this research successful. Also, I would like to thanks to all the reviewers for their insightful and valuable comment. This work was supported by the Deanship of Scientific Research (DSR), King Abdulaziz University, Saudi Arabia, under grant No. 830-129-D1437. Therefore, the author gratefully acknowledges the DSR technical and financial support.

\section{REFERENCES}

[1] T. Murakami, Y. Matsumoto, K. Fujii, and Y. Yamanaka, "Effects of multi-path propagation on microwave oven interference in wireless systems", IEEE International Symposium on Electromagnetic Compatibility, Vol. 2, pp 749-752, 2003

[2] T.W. Rondeau, M.F. .D'Souza, and D.G. Sweeney, "Residential microwave oven interference on Bluetooth data performance", IEEE Transactions on Consumer Electronics, Vol. 50, pp 856-863, 2004.

[3] I. F. Akyildiz, W. Lee, M. C. Vuran, and S. Mohanty. "Next generation/dynamic spectrum access/ cognitive radio wireless networks: A survey." Computer Networks, vol 50, pp. 2127-2159, 2006.

[4] S. Srikanteswara and C. Maciocco, "Interference mitigation using spectrum sensing," in Proc. Int. Conf. Comp. Comm. \& Net., pp. 39-44, 2007.

[5] S. Miyamoto, S. Harada, and N. Morinaga, "Performance of $2.4 \mathrm{GHz}$ band wireless LAN system using orthogonal frequency division multiplexing scheme under microwave oven noise environment," in Proc. IEEE Int. Symp. on Electromagn. Compat., vol. 1, pp. 157-162, 2005.
[6] A. Baid, S. Mathur, I. Seskar, T. Singh, S. Jain, D. Raychaudhuri, S. Paul, and A. Das, "Spectrum MRI: Towards diagnosis of multi-radio interference in the unlicensed band,"IEEE WCNC, 2011.

[7] Y. Matsumoto, M. Takeuchi, K. Fujii, A. Sugiura, and Y. Yamanaka, "Performance analysis of interference problems involving DS-SS WLAN systems and microwave ovens" IEEE Transactions on Electromagnetic Compatibility, Vol. 47, pp. 45-53, 2005

[8] P.S. Neelakanta, and J. Sivaraks, "A Novel Method to Mitigate Microwave Oven Dictaded EMI on Bluetooth Communications," Microwave Journal, pp. 70-88, 2001

[9] C.R. Buffler, and P.O. Risman, "Compatibility Issues between Bluetooth and High Power Systems in the ISM Band," Microwave Journal, pp. 126-131, 2000

[10] IEEE, "IEEE Std.802. 11b-1999 Wireless LAN medium access control and physical layer specifications: Higher-speed physical layer extension in the $2.4 \mathrm{GHz}$ band," IEEE Std. 802.11b, 1999.

[11] S. Yin, Z. Qu, and S. Li, "Achievable Throughput Optimization in Energy Harvesting Cognitive Radio Systems" IEEE Journal on Selected Areas in Communications, vol 33, 2015.

[12] C. S. Karthikeyan, and M. Suganthi, "Optimized Spectrum Sensing Algorithm for Cognitive Radio," Wireless Personal Communications, Vol 94, 2017.

[13] S. Shantanu, and S. A. Kumar, "On detecting termination in cognitive radio networks." International Journal of Network Management, vol 24, 2014.

[14] K. A. Ali, R. M. Husain, and R. Martin, "Cognitive Radio for Smart Grids: Survey of Architectures, Spectrum Sensing Mechanisms, and Networking Protocols" IEEE Communications Surveys \& Tutorials, vol 18, 2016.

[15] Y. Chungang, L. Jiandong, N. Qiang, A. Alagan, and G. Mohsen, "Interference-Aware Energy Efficiency Maximization in 5G UltraDense Networks" IEEE Transactions on Communications, vol 65, 2017.

[16] D. V. Son, N. V. Dinh, and S. O. Soon, "InterferenceAware Transmission for D2D Communications in a Cellular Network" Wireless Personal Communications, vol 98, 2018.

[17] G. Verma and O. P. Sahu, "Interference Aware Sensing Scheme in Cognitive Radio System" Wireless Personal Communications, vol 94, 2017.

[18] [18] K. Hyunsoon, and K.Hwangnam,"Designing interferenceaware network selection protocol for WLAN mobile devices" Transactions on Emerging Telecommunications Technologies, vol 28, 2017. 